\title{
Development of Alginate Microspheres Containing Chuanxiong for Oral Administration to Adult Zebrafish
}

\author{
Li-Jen Lin, ${ }^{1}$ Chung-Jen Chiang, ${ }^{2}$ Yun-Peng Chao, ${ }^{3,4}$ Shulhn-Der Wang, \\ Yu-Ting Chiou, ${ }^{3}$ Han-Yu Wang, ${ }^{3}$ and Shung-Te Kao ${ }^{1,6}$ \\ ${ }^{1}$ School of Chinese Medicine, College of Chinese Medicine, China Medical University, Taichung 40402, Taiwan \\ ${ }^{2}$ Department of Medical Laboratory Science and Biotechnology, China Medical University, Taichung 40402, Taiwan \\ ${ }^{3}$ Department of Chemical Engineering, Feng Chia University, Taichung 40724, Taiwan \\ ${ }^{4}$ Department of Health and Nutrition Biotechnology, Asia University, Taichung 41354, Taiwan \\ ${ }^{5}$ School of Post-Baccalaureate Chinese Medicine, College of Chinese Medicine, China Medical University, Taichung 40402, Taiwan \\ ${ }^{6}$ Department of Chinese Medicine, China Medical University Hospital, Taichung 40402, Taiwan
}

Correspondence should be addressed to Shung-Te Kao; stkao@mail.cmu.edu.tw

Received 26 January 2016; Accepted 29 May 2016

Academic Editor: Sung-Hoon Kim

Copyright (C) 2016 Li-Jen Lin et al. This is an open access article distributed under the Creative Commons Attribution License, which permits unrestricted use, distribution, and reproduction in any medium, provided the original work is properly cited.

Oral administration of Traditional Chinese Medicine (TCM) by patients is the common way to treat health problems. Zebrafish emerges as an excellent animal model for the pharmacology investigation. However, the oral delivery system of TCM in zebrafish has not been established so far. This issue was addressed by development of alginate microparticles for oral delivery of chuanxiong, a TCM that displays antifibrotic and antiproliferative effects on hepatocytes. The delivery microparticles were prepared from gelification of alginate containing various levels of chuanxiong. The chuanxiong-encapsulated alginate microparticles were characterized for their solubility, structure, encapsulation efficiency, the cargo release profile, and digestion in gastrointestinal tract of zebrafish. Encapsulation of chuanxiong resulted in more compact structure and the smaller size of microparticles. The release rate of chuanxiong increased for alginate microparticles carrying more chuanxiong in simulated intestinal fluid. This remarkable feature ensures the controlled release of encapsulated cargos in the gastrointestinal tract of zebrafish. Moreover, chuanxiong-loaded alginate microparticles were moved to the end of gastrointestinal tract after oral administration for $6 \mathrm{hr}$ and excreted from the body after $16 \mathrm{hr}$. Therefore, our developed method for oral administration of TCM in zebrafish is useful for easy and rapid evaluation of the drug effect on disease.

\section{Introduction}

Chuanxiong is a Traditional Chinese Medicine (TCM) herb and also a common dietary in Asia. It contains more than 200 isolated compounds, and tetramethylpyrazine is identified as one of the most active ingredients $[1,2]$. The high medicinal value of chuanxiong can be acknowledged by its ability to promote blood circulation and alleviate pain [3]. This herb has been widely used in treatment of cardiovascular disease, atherosclerosis [4], and thrombus formation [5]. In particular, chuanxiong exhibits the antifibrotic and antiproliferative effects on hepatocytes as reported by in vitro and in vivo studies $[6,7]$.

Chronic hepatitis B virus (HBV) and C virus (HCV) infections are usually associated with the progression of hepatic fibrosis [8]. HBV and HCV are involved in the formation of intrahepatic cholangiocarcinoma (ICC), and coinfection of both viruses increases the severity of hepatitis and hepatic fibrosis $[8,9]$. ICC is the second most common hepatic malignancy with poor prognosis and a low resectability rate [8]. To study this hepatic disease, the transgenic model of ICC has been developed by coexpression of HBV X and HCV core protein in zebrafish [8]. This model displays similar histopathology and molecular features including neoplasm networks and potential biomarkers to human ICC. Apparently, it facilitates the investigation on the effect of therapeutic drugs on HBV- and HCV-induced fibrosis and bile duct neoplasms.

Zebrafish is recognized as an excellent animal model for gene studies and toxicity analyses of vertebrates. In 
particular, the human disease states are usually mirrored by the mutant phenotypes of zebrafish. Therefore, the development of transgenic zebrafish models for human diseases offers a promising means to investigate the corresponding pathophysiology and screen potential therapeutic drugs [10]. To analyze the drug effects, adult zebrafish are conventionally given medication by direct bathing the drugs in a chemical reagent or by peritoneal injection of the drugs. Note that TCM is administrated orally by human patients. It is obvious that a new delivery system of TCM is required for studies of their effects in the zebrafish models. This issue was addressed by using alginate for oral delivery of chuanxiong. Alginate is a polymer with high biological compatibility and has been used for encapsulation of drugs, proteins, and cells [11-13]. In this study, the alginate-based delivery system was developed for oral administration of chuanxiong in zebrafish. Our results show that this delivery system has a potential for rapid selection of TCM for effective treatment of diseases.

\section{Materials and Methods}

2.1. Zebrafish and Reagents. The experiments using the zebrafish $\mathrm{AB}$ strain were carried out in agreement with the principles outlined by the Committee of China Medical University (Number 103-232-C). The zebrafish strain was maintained in light cycle of $14 \mathrm{hr}$ light $/ 10 \mathrm{hr}$ dark at $25^{\circ} \mathrm{C}$. Chuanxiong (Kaiser Pharmaceutical Co., Ltd., Taiwan) was dissolved in distilled water for encapsulation by alginate microparticles and stored at $-20^{\circ} \mathrm{C}$ until use. Alginate and TetraBits ${ }^{\circledR}$ Complete (fish food) were purchased from Chuang Song Zong Pharmaceutical Co., Ltd. (Kaohsiung, Taiwan) and Tetra GmbH (Germany), respectively.

2.2. Encapsulation of Chuanxiong by Alginate Microparticles. The dose of chuanxiong is based on the doctors' prescriptions for liver cancer treatment in Taiwan. A daily dose for an adult is $1 \mathrm{x}$ chuanxiong $(14.29 \mathrm{mg} / \mathrm{kg})$. Alginate microparticles were prepared by dropwise addition of the alginate solution $(15 \mathrm{~mL})$ containing $1 \%$ alginate and $1.5 \mathrm{~g}$ of fish food into $100 \mathrm{~mL}$ calcium chloride (1.5\%) solution with magnetic stirring. Microparticles were formed and then left in the solution for $10 \mathrm{~min}$ at room temperature. Similarly, the chuanxiongloaded alginate microparticles were prepared using the alginate solution containing $0.5 \mathrm{x}, 1 \mathrm{x}$, or $1.5 \mathrm{x}$ chuanxiong. The sizes of alginate microparticles free of or encapsulated with chuanxiong were determined by optical microscope system (Carl Zeiss Axioskop 2, Germany).

2.3. Characterization of Alginate Microparticles. The swelling behavior of free or chuanxiong-loaded alginate microparticles was investigated by soaking dried ones into $200 \mu \mathrm{L}$ water or simulated intestinal fluid ( $\mathrm{pH}$ 6.8) in the 96-well plate for $24 \mathrm{hr}$. The composition of simulated intestinal fluid contained $6.8 \mathrm{~g}$ of monobasic potassium phosphate and $0.2 \mathrm{~N} \mathrm{NaOH}$ $(190 \mathrm{~mL})$ per L. Microparticles were sampled and their sizes were then determined at time intervals. The swelling rate of microparticles was calculated as follows:

$$
\text { Swelling rate }=\frac{(\text { diameter of swollen microparticle }- \text { diameter of dried microparticle })}{\text { diameter of dried microparticle }} \text {. }
$$

In addition, the morphology of alginate microparticle was examined by scanning electron microscopy (SEM). Followed by dehydration using Critical Point Dryer (CPD), one microparticle was attached to aluminum stubs and examined in a field emission scanning electron microscope (JEOL JSM7401F). Moreover, one free or loaded alginate microparticle was clamped and its size was measured. The shape change of microparticle was calculated as follows:

$$
\text { Shape change rate }=\frac{(\text { diameter of clamped microparticle }- \text { diameter of microparticle })}{\text { diameter of microparticle }} \times 100 \% \text {. }
$$

2.4. In Vitro Release Study of Alginate Microparticles. The in vitro release study was carried out with the chuanxiongloaded alginate microparticles in water and simulated intestinal fluid. Microparticles $(0.2 \mathrm{~g})$ were placed in a $15 \mathrm{~mL}$ tube containing $2 \mathrm{~mL}$ of water or simulated intestinal fluid and incubated at room temperature with shaking at $100 \mathrm{rpm}$. The solution $(0.5 \mathrm{~mL})$ was sampled for determination of released chuanxiong by measuring ferulic acid using HPLC at time intervals. HPLC was equipped with Waters 515 HPLC Pump, Waters 2996 Photodiode Array Detector (PDA), and Waters SunFire ${ }^{\mathrm{TM}} \mathrm{C} 185 \mu \mathrm{m} 4.6 \times 250 \mathrm{~mm}$ column. The mobile phase consisted of $2 \%$ acetic acid in water/acetonitrile and the flow rate was set at $0.6 \mathrm{~mL} / \mathrm{min}$. A gradient elution was performed with $0-100 \%$ acetonitrile in $0 \sim 50 \mathrm{~min}$ and $100-0 \%$ acetonitrile in 55 60 min. The eluate was detected at $320 \mathrm{~nm}$. The encapsulation and release efficiency of chuanxiong by alginate microparticles were calculated as follows:

$$
\begin{aligned}
& \text { Encapsulation efficiency } \\
& \qquad=\left(\frac{\text { loaded chuanxiong }}{\text { original chuanxiong }}\right) \times 100 \%,
\end{aligned}
$$




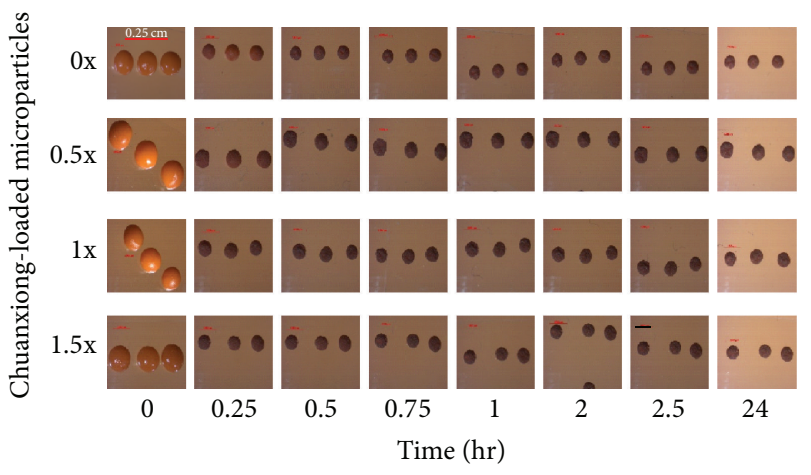

(a)

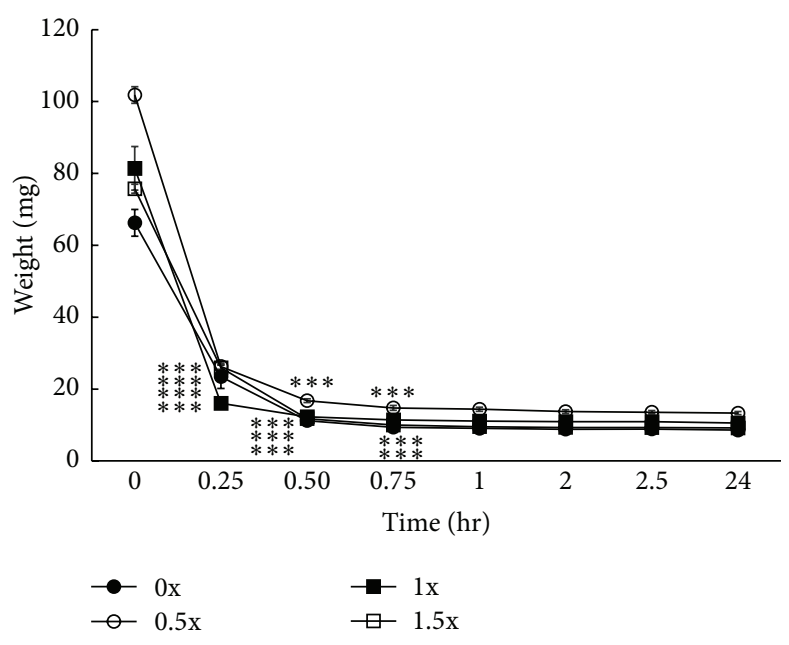

(b)

FIGURE 1: The effect of drying on the size and weight of chuanxiong-loaded alginate microparticles. (a) The size of microparticles free of (0x) or encapsulated with chuanxiong. The time before drying was denoted by "0." (b) The weight of microparticles free of or encapsulated with chuanxiong. Values represent the mean \pm SD of 10 microparticles per group. ${ }^{* * *} P<0.001$ (between after time and before time in the same group).

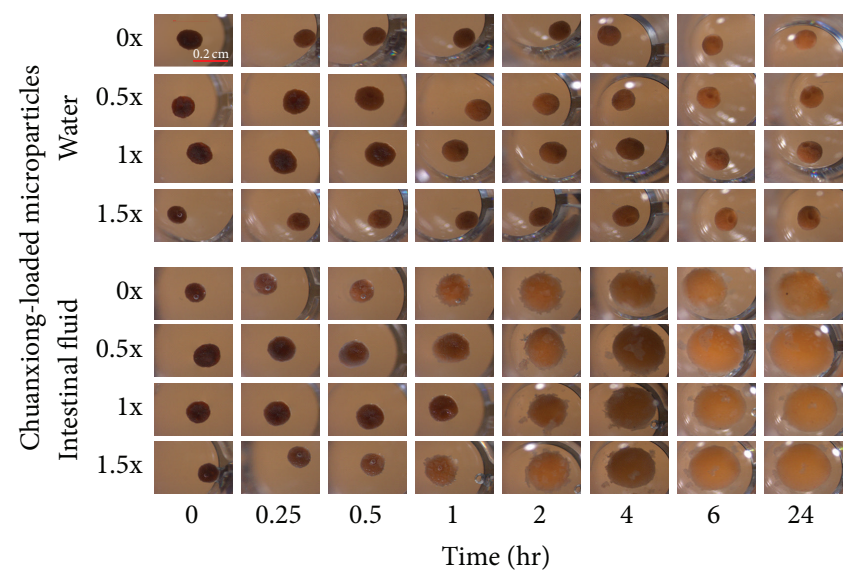

(a)

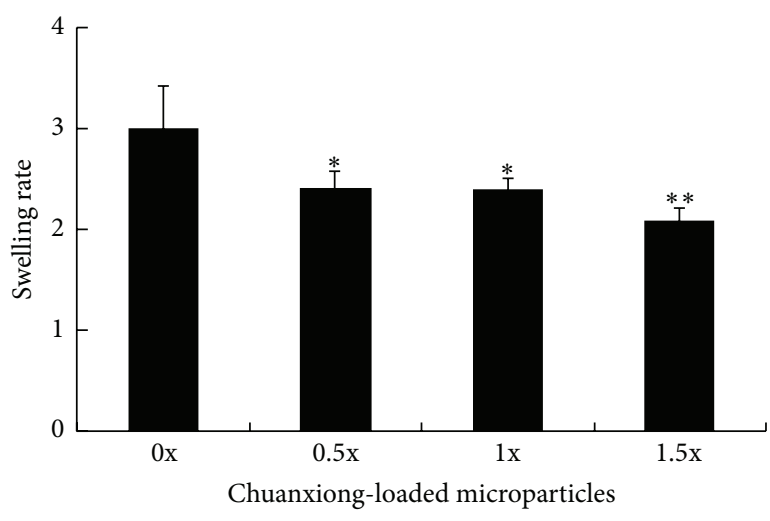

(b)

Figure 2: The swelling behavior of chuanxiong-loaded alginate microparticles. (a) The size of alginate microparticles free of or encapsulated with chuanxiong in water and simulated intestinal fluid. (b) The swelling rate of microparticles were compared with the same microparticle size between 24 hours and 0 mins in simulated intestinal fluid. Values represent the mean $\pm \mathrm{SD} .{ }^{*} P<0.05$; ${ }^{* *} P<0.01$ (versus $0 \mathrm{x}$ group).

$$
\text { Release efficiency }=\left(\frac{\text { released chuanxiong }}{\text { loaded chuanxiong }}\right) \times 100 \%
$$

2.5. Oral Administration of Alginate Microparticles. Adult zebrafish were fasted overnight and randomly placed into tanks (one fish per tank) before oral administration of alginate microparticles which contained red dye (Widetex Co., Taiwan). Each fish was sacrificed at $0,1,3,6,9$, and $16 \mathrm{~h}$ ( $n=6$ at each point $)$ after oral administration. The gastrointestinal tract of fish was observed by optical microscope system (Carl Zeiss Axioskop 2, Germany) and fluorescence microscope (OLYMPUS, BX50, Japan) to assess consumption of microparticles.
2.6. Statistics. Data were reported as mean \pm standard deviation (SD). The statistical analysis was analyzed by Student's $t$-test, and $P$ value $<0.05$ indicated the statistical significance.

\section{Results}

3.1. Characteristic of Chuanxiong-Encapsulated Alginate Microparticles. Alginate microparticles free of or encapsulated with various chuanxiong were prepared for studies. To facilitate the feeding of zebrafish, these microparticles were reshaped by drying in an incubator at $37^{\circ} \mathrm{C}$. The sizes of microparticles were reduced by half after drying for $0.25 \mathrm{~h}$ and gradually shank from $0.25 \mathrm{~h}$ to $0.5 \mathrm{~h}$ (Figure 1(a)). After that, the size and the weight of microparticles remained 

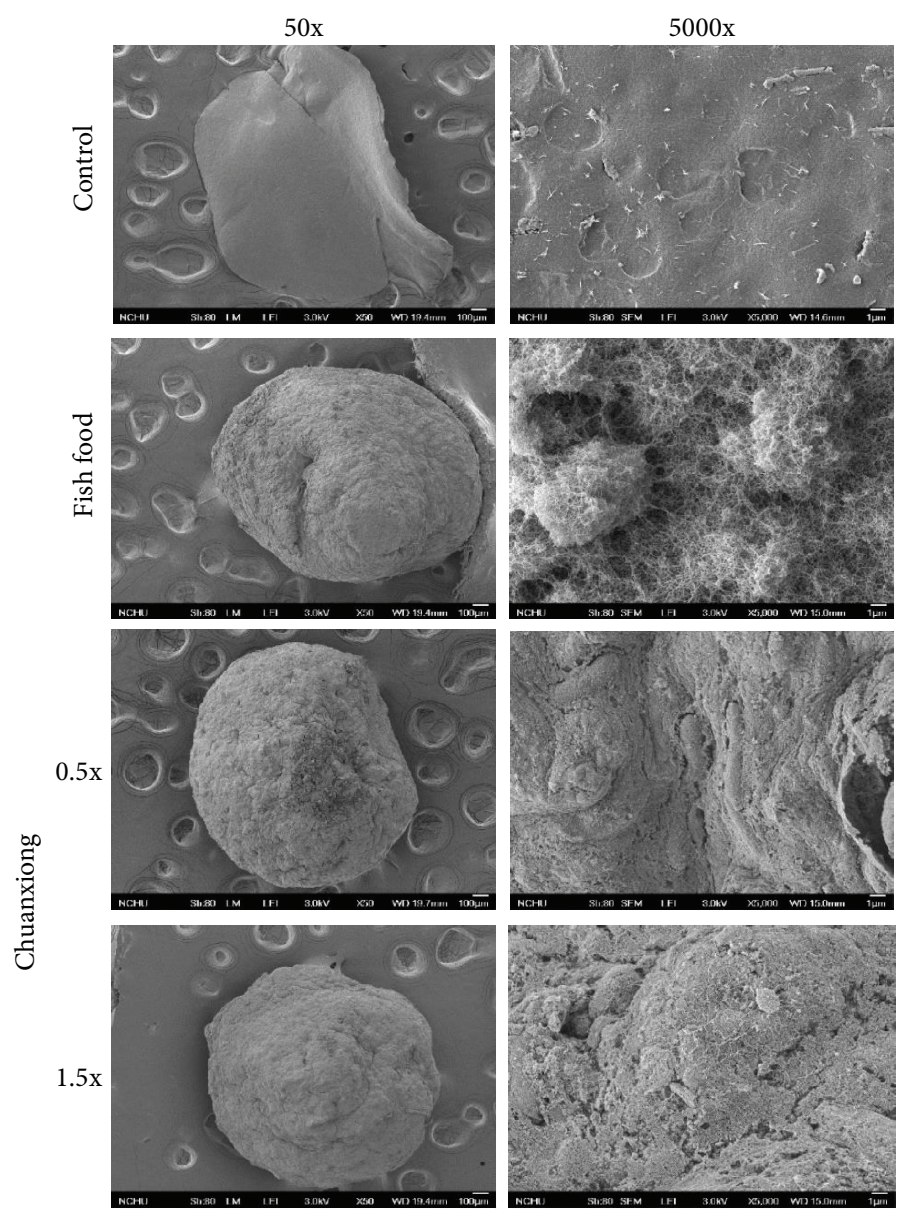

(a)
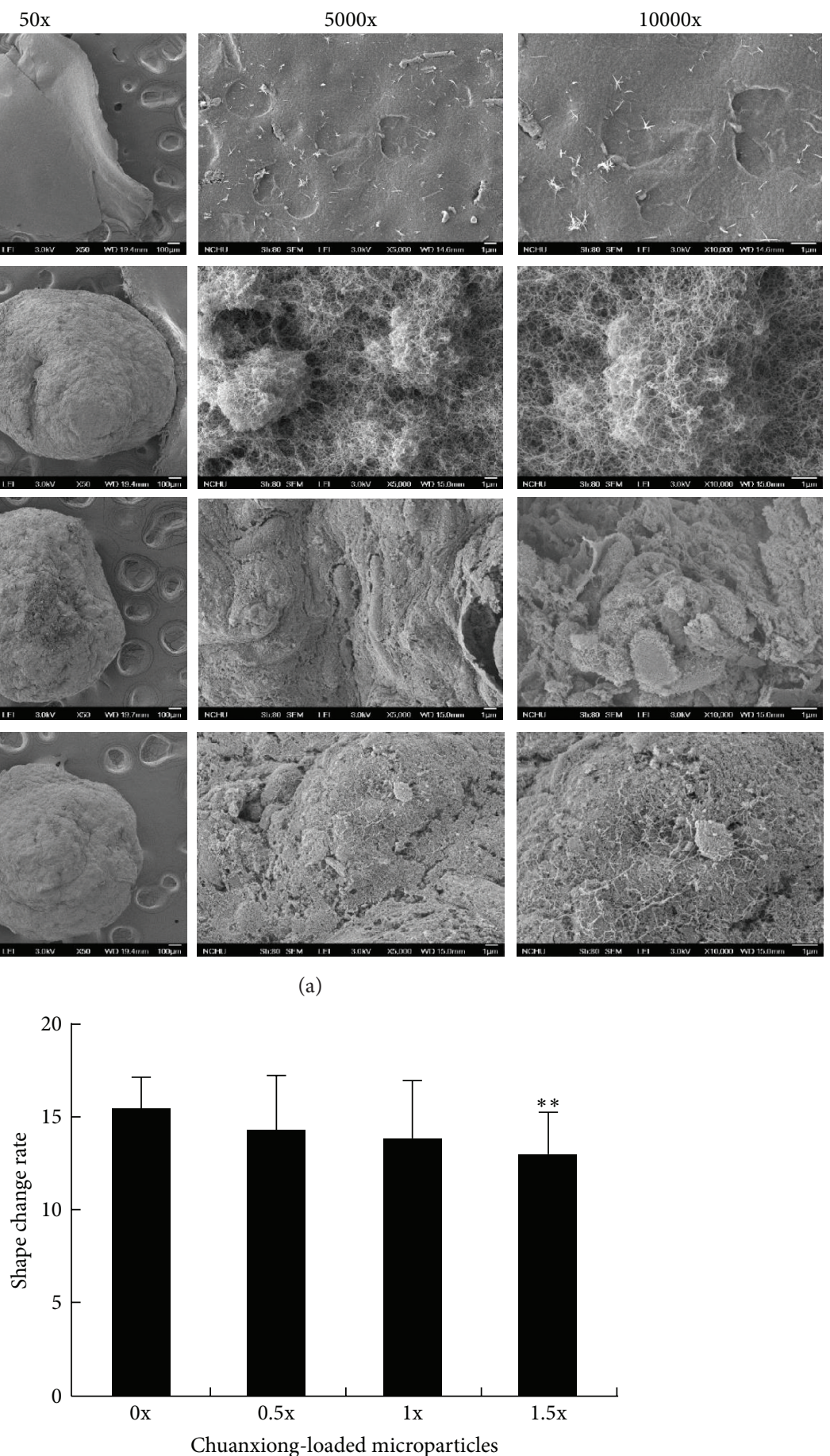

(b)

FIGURE 3: The effect of chuanxiong on the structure of alginate microparticles. (a) The SEM analysis with amplification at 50x, 5000x, and $10000 x$. (b) The shape change rate of microparticles free of or encapsulated with chuanxiong. Values represent the mean \pm SD. ${ }^{* *} P<0.01$ (versus 0x group).

almost unchanged. Dried microparticles exhibited the biggest size and the heaviest weight among all (Figure 1(b)).

Dried microparticles were soaked into water and stimulated intestinal fluid. No swelling for these microparticles was observed in water (Figure 2(a)). In contrast, microparticles swelled in the simulated intestinal fluid and their sizes increased along the time course. The size of microparticles free of chuanxiong was increased by 3 -fold while the size of chuanxiong-encapsulated microparticles was approximately doubled (Figure 2(b)). 
TABLE 1: Characteristics of chuanxiong-loaded alginate microparticles. Values represent the mean \pm SD. ${ }^{*} P<0.05 ;{ }^{* *} P<0.01$; and ${ }^{* * *} P<$ 0.001 (versus $0.5 x$ group).

\begin{tabular}{lccccc}
\hline $\begin{array}{l}\text { Chuanxiong- } \\
\text { loaded } \\
\text { microparticle }\end{array}$ & $\begin{array}{c}\text { Encapsulation } \\
\text { efficiency\% }\end{array}$ & $\begin{array}{c}\text { Release } \\
\text { efficiency\% }\end{array}$ & $\begin{array}{c}\text { Drug loading } \\
\text { Chuanxiong } \\
(\mu \mathrm{g} / \text { microparticle) }\end{array}$ & $\begin{array}{c}\text { Minima } \\
\text { (microparticles/day) }\end{array}$ & $\begin{array}{c}\text { Daily dosage } \\
\text { (microparticles/day) }\end{array}$ \\
\hline $0.5 \mathrm{x}$ & $76.72 \pm 1.19$ & $9.88 \pm 0.54$ & $8.70 \pm 0.47$ & $1.57 \pm 0.08$ & $2.35 \pm 0.12$ \\
$1 \mathrm{x}$ & $73.35^{* *} \pm 0.15$ & $12.74^{* * *} \pm 0.23$ & $23.09^{* * *} \pm 0.42$ & $1.18 \pm 0.02$ & $1.77 \pm 0.03$ \\
$1.5 \mathrm{x}$ & $74.29^{*} \pm 0.34$ & $11.50^{*} \pm 0.44$ & $29.22^{* * *} \pm 1.11$ & $1.40 \pm 0.05$ & $2.10 \pm 0.08$ \\
\hline
\end{tabular}

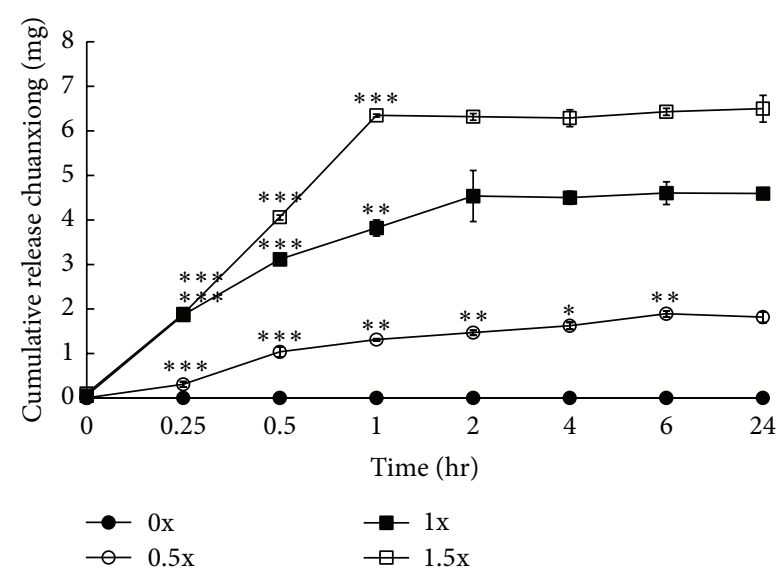

FIGURE 4: The release efficiency of chuanxiong-loaded alginate microparticles. Values represent the mean $\pm \mathrm{SD} .{ }^{*} P<0.05 ;{ }^{* *} P<$ 0.01 ; and ${ }^{* * *} P<0.001$ (between after time and before time in the same group).

\subsection{Morphology of Chuanxiong-Encapsulated Alginate Micro-} particles. The effect of encapsulated chuanxiong on microparticles structure was investigated by SEM. In comparison with pure alginate microparticles, microparticles loaded with fish food assumed a loose structure as revealed by the SEM analysis (Figure 3(a)). The additional encapsulation of chuanxiong rendered the structure of microparticle more compact. In addition, the shape change rate of alginate microparticles decreased with the increasing level of encapsulated chuanxiong (Figure 3(b)). Based on the definition of the shape change rate, the result indicates that the structure of alginate microparticles after encapsulation with chuanxiong becomes more stretchable.

3.3. In Vitro Release Profiles. The release profile of chuanxiong-encapsulated alginate microparticles was studied in vitro. As shown in Figure 4, chuanxiong was released from alginate microparticles along the time course. Microparticles with $1.5 x$ chuanxiong exhibited the most rapid release of the herb cargo compared to others. There was $11.5 \%$ of the loaded chuanxiong (ca. $29.22 \mu \mathrm{g} /$ macroparticle) released from $1.5 \mathrm{x}$ chuanxiong-encapsulated microparticles at $24 \mathrm{~h}$ (Table 1). Interestingly, microparticles with $0.5 x$ chuanxiong exhibited a prolonged release profile. Moreover, the encapsulation efficiency of chuanxiong-loaded microparticles reached $76.72 \%$ $74.29 \%$. As estimated, oral administration of 2 microparticles containing $0.5 \mathrm{x}, 1 \mathrm{x}$, or $1.5 \mathrm{x}$ chuanxiong per day for one fish could reach the maximum dosage of chuanxiong (Table 1).

3.4. Oral Administration of Chuanxiong-Encapsulated Alginate Microparticles. Finally, oral administration of alginate microparticles was carried out in the $\mathrm{AB}$ line zebrafish twice a day (in the morning and afternoon). It was found that the average time for uptake of one microparticle with no, $0.5 \mathrm{x}$, $1 \mathrm{x}$, and $1.5 \mathrm{x}$ chuanxiong by a single fish was $47,23,31$, and 34 seconds $(n=40)$, respectively. Moreover, the gastrointestinal tract of zebrafish was observed after administration of the $1.5 \mathrm{x}$ chuanxiong-loaded microparticles (Figure 5). Microparticles were digested in chylomicrons after $9 \mathrm{~h}$ and excreted from the body after $16 \mathrm{~h}$.

\section{Discussion}

Carriers such as micelles, dendrimers, and microspheres are commonly employed for the oral delivery of drugs, and the receptor-, paracellular-, carrier-, $M$ cell-, or/and transcellular-mediated adsorption of the loaded drugs proceed in intestinal epithelium [14]. The carrier materials usually involve chitosan, alginate, PLA, and PGA which are biodegradable as well as biocompatible and approved for human use by FDA $[14,15]$. Moreover, these polymeric materials have flexibility in size, surface charge, and hydrophobicity and are endowed with the property for controlled release of drug [15]. In this study, the oral administration system of TCM in zebrafish was developed based on alginate. This was illustrated by encapsulation of chuanxiong with alginate. The resulting alginate microparticles were dried and utilized as fish feed. Dried alginate microparticles were insoluble in water and became swollen in simulated intestinal fluid (Figure 2). This remarkable feature ensures the controlled release of encapsulated drugs in the gastrointestinal tract of zebrafish. Loaded with more chuanxiong, alginate microparticles after drying had a smaller swollen size in stimulated intestinal fluid. It is likely that interaction of chuanxiong compositions with alginate results in more compact structure of microparticles. This view is supported by the SEM analysis, revealing that encapsulation of chuanxiong leads to more compact structure and the smaller size of microparticles (Figure 3).

The release of chuanxiong from alginate microparticles is unaffected by their compact structure. As shown in Figure 4, the release rate of chuanxiong increased for alginate microparticles carrying more chuanxiong and the 

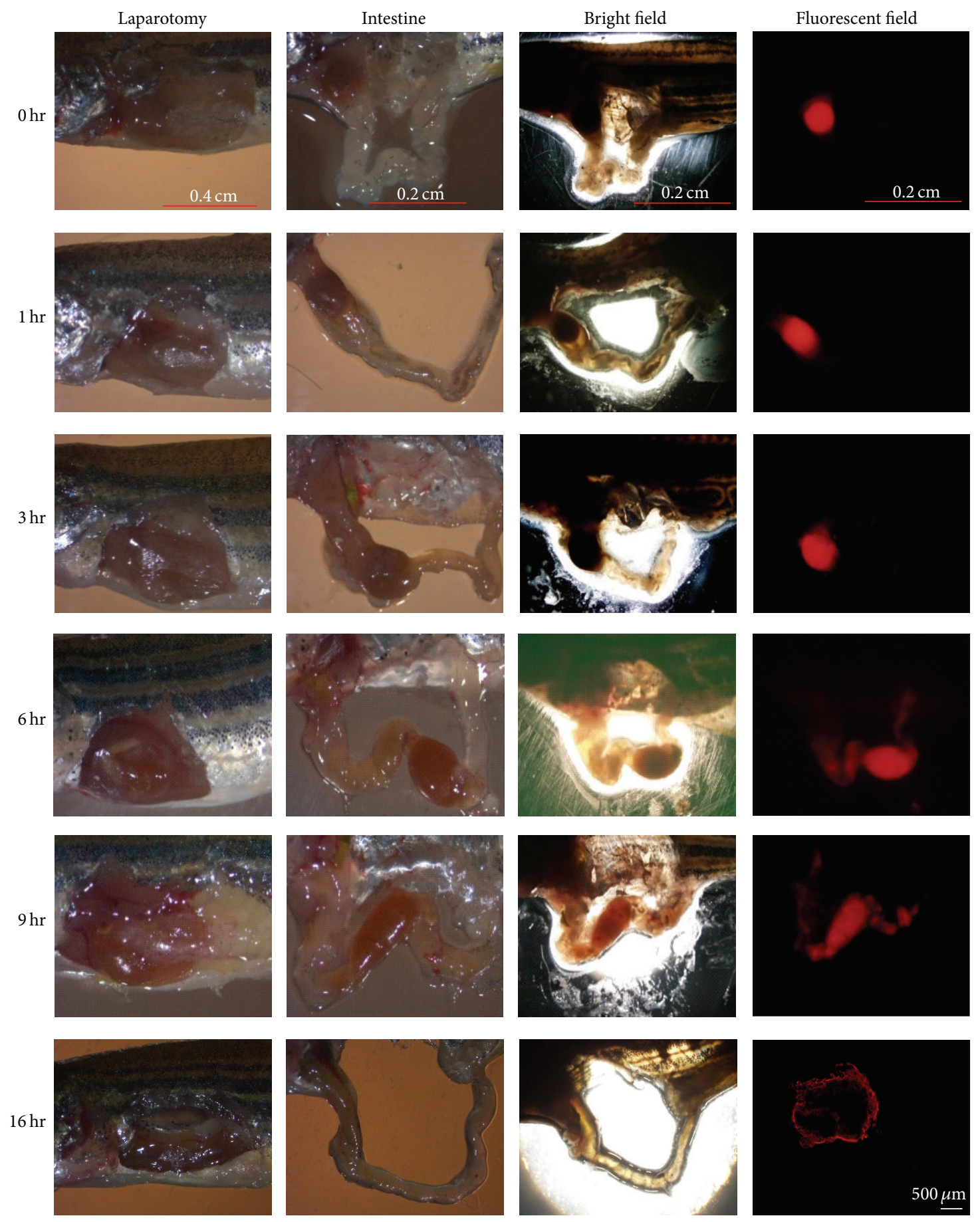

FIGURE 5: The analysis of chuanxiong-loaded alginate microparticles in zebrafish intestine. The microparticles were excreted after $16 \mathrm{~h}$ as shown in the fluorescent field.

released chuanxiong from microparticles with $0.5 \mathrm{x}, 1 \mathrm{x}$, and $1.5 \mathrm{x}$ chuanxiong almost reached the plateau. It was estimated that 1.5 and 2 particles per day for one zebrafish achieved the minimum and maximum dosage for one patient according to the release profiles of alginate microparticles loaded with 3 levels of chuanxiong. Moreover, alginate microparticles were moved to the end of gastrointestinal tract after oral administration for $6 \mathrm{hr}$, digested in chylomicrons after $9 \mathrm{hr}$, and excreted from the body after $16 \mathrm{hr}$. Therefore, the encapsulated chuanxiong released from the ingested alginate microparticles could reach the effective dosage before being excreted. This model offers an advantage of the study on the disease treatment by TCM in zebrafish.

Cancer has become the most life-threatening illness worldwide. There are around 14.1 million diagnosed cases of cancer and 8.2 million people who die of cancers per 
year [16]. Apparently, it is important to have an efficient method for evaluation of the drug efficacy in cancer therapy. Most clinical trials have shown the usefulness of TCM for disease/cancer treatment [17-19]. In addition, over $70 \%$ of zebrafish genes share similarity with human genes [20] and zebrafish cancers are also similar to human cancers in the histological and genetic aspects [21]. We developed a new method to prepare alginate microparticles for oral delivery of chuanxiong (a TCM) which were stable and attractive to zebrafish to eat easily. The cumulative release chuanxiong of two chuanxiong-encapsulated alginate microparticles could reach clinical daily dosage before $6 \mathrm{hr}$ in simulated intestinal fluid. It not only solves the oral administration zebrafish with TCM but also provides new field to explore therapy mechanism of TCM by disease model of transgenic zebrafish. Therefore, this technique may contribute to development of new drugs that are more suited for modern people.

\section{Competing Interests}

All authors declare no conflict of interests.

\section{Acknowledgments}

This work was supported by CMU under the Aim for Top University Plan of the Ministry of Education, Taiwan.

\section{References}

[1] X. Xie, Y. Tian, S. Yin, Y. Lin, and G. Tan, "Anticancer effects of Ligusticum chuanxiong Hort alcohol extracts on HS766T cell," African Journal of Traditional, Complementary, and Alternative Medicines, vol. 10, no. 6, pp. 542-546, 2013.

[2] W. Li, Y. Tang, Y. Chen, and J.-A. Duan, "Advances in the chemical analysis and biological activities of chuanxiong," Molecules, vol. 17, no. 9, pp. 10614-10651, 2012.

[3] X. M. Gao, Traditional Chinese Pharmacy, China Press of Traditional Chinese Medicine, Beijing, China, 2006.

[4] L. Zhang, Y.-R. Jiang, C.-Y. Guo, C.-F. Wu, K.-J. Chen, and H.-J. Yin, "Effects of active components of Red Paeonia and Rhizoma chuanxiong on angiogenesis in atherosclerosis plaque in rabbits," Chinese Journal of Integrative Medicine, vol. 15, no. 5, pp. 359-364, 2009.

[5] M. Li, S. Handa, Y. Ikeda, and S. Goto, "Specific inhibiting characteristics of tetramethylpyrazine, one of the active ingredients of the Chinese herbal medicine 'Chuanxiong,' on platelet thrombus formation under high shear rates," Thrombosis Research, vol. 104, no. 1, pp. 15-28, 2001.

[6] Y.-L. Lin, Y.-C. Hsu, Y.-T. Chiu, and Y.-T. Huang, "Antifibrotic effects of a herbal combination regimen on hepatic fibrotic rats," Phytotherapy Research, vol. 22, no. 1, pp. 69-76, 2008.

[7] Y.-L. Lin, T.-F. Lee, Y.-J. Huang, and Y.-T. Huang, "Inhibitory effects of Ligusticum chuanxiong on the proliferation of rat hepatic stellate cells," Journal of Gastroenterology and Hepatology, vol. 21, no. 8, pp. 1257-1265, 2006.

[8] W. Liu, J.-R. Chen, C.-H. Hsu et al., "A zebrafish model of intrahepatic cholangiocarcinoma by dual expression of hepatitis $\mathrm{B}$ virus $\mathrm{X}$ and hepatitis $\mathrm{C}$ virus core protein in liver," Hepatology, vol. 56, no. 6, pp. 2268-2276, 2012.
[9] Z. Liu and J. Hou, "Hepatitis B virus (HBV) and hepatitis C virus (HCV) dual infection," International Journal of Medical Sciences, vol. 3, no. 2, pp. 57-62, 2006.

[10] L. Zang, D. Morikane, Y. Shimada, T. Tanaka, and N. Nishimura, "A novel protocol for the oral administration of test chemicals to adult zebrafish," Zebrafish, vol. 8, no. 4, pp. 203-210, 2011.

[11] A. Sosnik, "Alginate particles as platform for drug delivery by the oral route: state-of-the-art," ISRN Pharmaceutics, vol. 2014, Article ID 926157, 17 pages, 2014.

[12] K. Ghosal, A. Ranjan, and B. B. Bhowmik, "A novel vaginal drug delivery system: anti-HIV bioadhesive film containing abacavir," Journal of Materials Science: Materials in Medicine, vol. 25, no. 7, pp. 1679-1689, 2014.

[13] R. Guo, L. Chen, S. Cai et al., "Novel alginate coated hydrophobically modified chitosan polyelectrolyte complex for the delivery of BSA," Journal of Materials Science: Materials in Medicine, vol. 24, no. 9, pp. 2093-2100, 2013.

[14] U. Agrawal, R. Sharma, M. Gupta, and S. P. Vyas, "Is nanotechnology a boon for oral drug delivery?" Drug Discovery Today, vol. 19, no. 10, pp. 1530-1546, 2014.

[15] E. M. Pridgen, F. Alexis, and O. C. Farokhzad, "Polymeric nanoparticle technologies for oral drug delivery," Clinical Gastroenterology and Hepatology, vol. 12, no. 10, pp. 1605-1610, 2014.

[16] L. A. Torre, F. Bray, R. L. Siegel, J. Ferlay, J. Lortet-Tieulent, and A. Jemal, "Global cancer statistics, 2012," CA: A Cancer Journal for Clinicians, vol. 65, no. 2, pp. 87-108, 2015.

[17] X. Li, G. Yang, Y. Zhang et al., "Traditional Chinese medicine in cancer care: a review of controlled clinical studies published in chinese," PLoS ONE, vol. 8, Article ID e60338, 2013.

[18] M. Jiang, J. Yang, C. Zhang et al., "Clinical studies with traditional Chinese medicine in the past decade and future research and development," Planta Medica, vol. 76, no. 17, pp. 2048-2064, 2010.

[19] J. Liu, X. Li, J. Liu, L. Ma, X. Li, and V. Fønnebø, “Traditional chinese medicine in cancer care: a review of case reports published in Chinese literature," Forschende Komplementarmedizin, vol. 18, no. 5, pp. 257-263, 2011.

[20] K. Howe, M. D. Clark, C. F. Torroja et al., "The zebrafish reference genome sequence and its relationship to the human genome," Nature, vol. 496, no. 7446, pp. 498-503, 2013.

[21] R. White, K. Rose, and L. Zon, "Zebrafish cancer: the state of the art and the path forward," Nature Reviews Cancer, vol. 13, no. 9, pp. 624-636, 2013. 

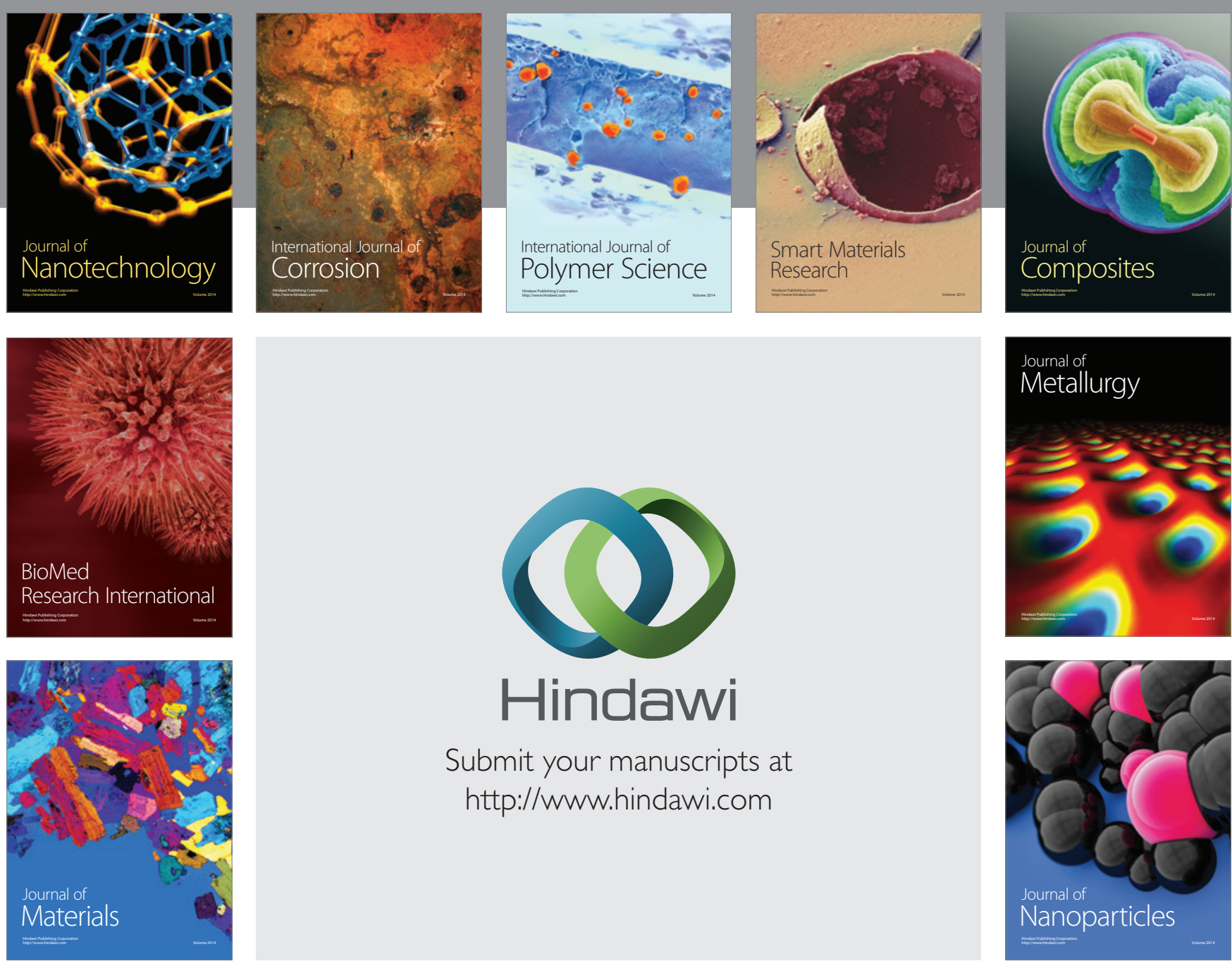

\section{Hindawi}

Submit your manuscripts at

http://www.hindawi.com

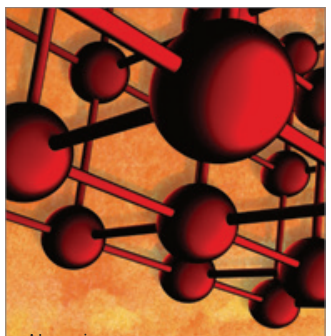

Materials Science and Engineering
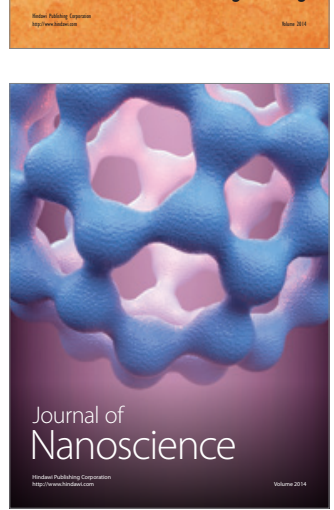
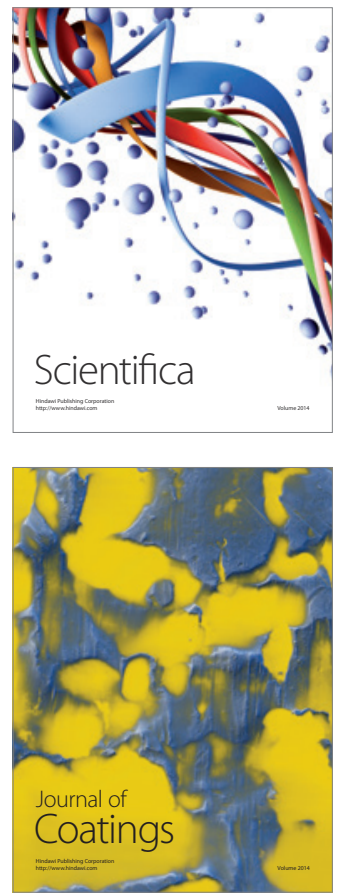
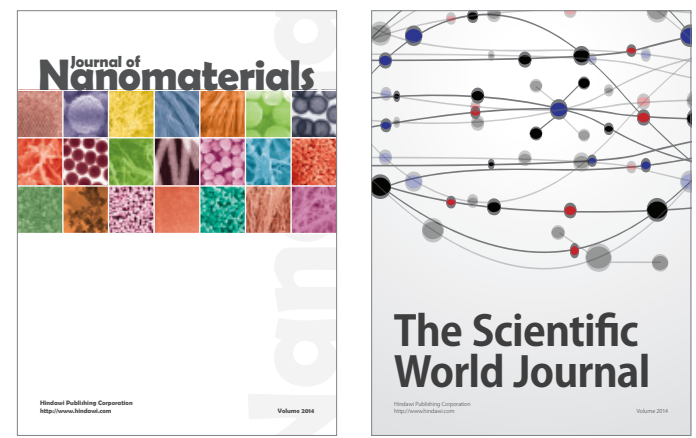

The Scientific World Journal
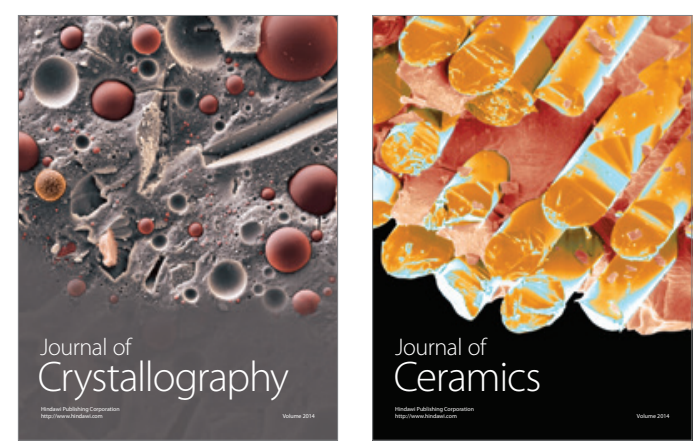
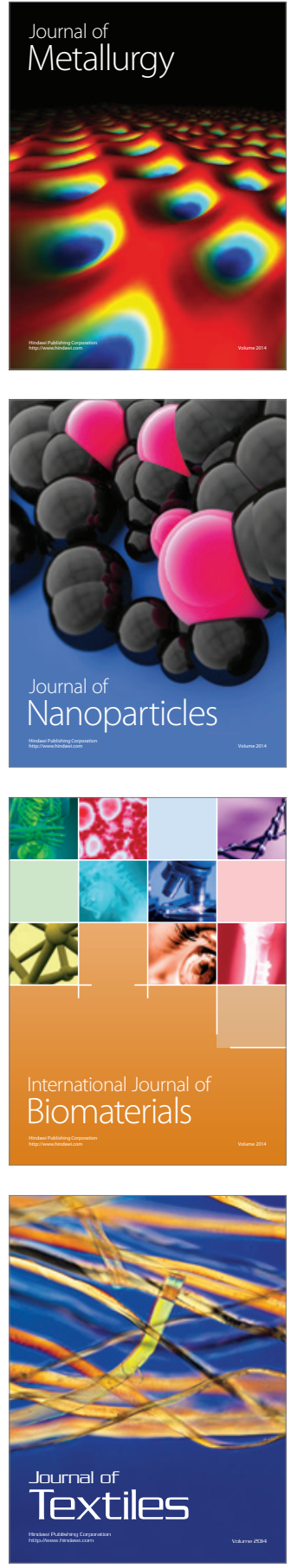